\title{
The role of pre- and post-SRS systemic therapy in patients with NSCLC brain metastases
}

\author{
Shyamal Patel' ${ }^{1}$, Waleed F Mourad1, Rajal Patel' ${ }^{1}$ Rafi Kabarriti1, Rebekah Young1, \\ Ravi Yaparpalvi', Linda Hong1, Patrick LaSala², Chandan Guha1, \\ Shalom Kalnicki' ${ }^{1}$, Madhur K Garg1 \\ ${ }^{1}$ Department of Radiation Oncology, Montefiore Medical Center, Bronx, New York, USA \\ 2Department of Neurosurgery, Montefiore Medical Center, Bronx, New York, USA
}

Received September 07, 2015; Revised November 28, 2015; Accepted November 30, 2015; Published Online December 07, 2015

Original Article

\begin{abstract}
Purpose: We report our experience with stereotactic radiosurgery (SRS) for NSCLC brain metastases. We then assess the prognostic value of pre- and post-SRS systemic therapy (PrSST and PoSST) and evaluate the timing of PoSST. Methods: In this retrospective study, we analyzed 96 patients with lung cancer and ECOG PS $\leq 3$ who underwent SRS during 2007-2013. Recorded factors included SRS treatment parameters, systemic status of disease (SDS) at time of SRS, and the use of PrSST and PoSST. SDS was designated as pulmonary disease or extrapulmonary disease. For analysis, the SRS-PoSST interval (SPI) was divided into $\leq 30$ days and $>30$ days. Univariate and multivariate analyses were performed. Results: 85 patients with NSCLC were included in this analysis. $48 \%$ received PrSST and $48 \%$ received PoSST. 57\% of patients had pulmonary disease while $40 \%$ had extrapulmonary disease. $46 \%$ of patients had synchronous metastases. At a median follow-up of 6 months, the median survival was 6.4 months and the actuarial overall survival at 3 , 6,12 , and 36 months was $80 \%, 52 \%, 31 \%$, and $6 \%$. Extrapulmonary disease $(\mathrm{p}=$ 0.008 ) negatively predicted for survival while the receipt of any systemic therapy $(\mathrm{p}=0.050)$ or PoSST alone $(\mathrm{p}=0.039)$ positively predicted for survival. In patients receiving PoSST, an SPI $>30$ days positively predicted for survival (HR $0.28,95 \% \mathrm{CI}$ $0.13-0.62, p=0.002$ ) regardless of SDS. Conclusion: Our results indicate the prognostic importance of systemic therapy and specifically PoSST. Additionally, delaying the initiation of PoSST to $>30$ days seems beneficial. This finding was potentially influenced by neurotoxicity after SRS. Further investigation is warranted to define the optimal SPI.
\end{abstract}

Keywords: SRS and Chemotherapy; Lung Cancer Brain Metastases; SRS Chemotherapy Interval; SRS for NSCLC Brain Metastases

\section{Introduction}

It is estimated that $170-200,000$ new cases of brain metastases are diagnosed each year in the United States $^{1}$, and lung cancer remains as the predominant source accounting for up to $50 \%$ of cases. Of patients with lung cancer, at least $19 \%$ will develop brain metastases. $^{2}$

The traditional treatment for brain metastases entailed the use whole brain radiation therapy (WBRT). Patchell et al. demonstrated a survival benefit with the addition of metastectomy prior to WBRT, and subsequently found an improvement in locoregional control with the addition of WBRT to resection. ${ }^{3,4}$ With the advent of newer radiation technology, the utilization of more focal therapy - stereotactic radiosurgery (SRS) - has gained more traction in addressing brain metastases. While randomized controlled trials have shown that SRS can be used in addition to ${ }^{5}$ or instead of ${ }^{6,7}$ WBRT, the addition of WBRT to SRS continues to depend on institutional bias and patient selection criteria in an attempt to balance intracranial control with preservation of cognitive function. 
The use of systemic therapy (chemotherapy or targeted therapies) in patients with brain metastases from lung cancer has also been examined.8,13 While the effectiveness of systemic therapy in patients with brain metastases has been questioned, some studies have demonstrated an intracranial response after systemic agents in patients with or without WBRT. ${ }^{14,16}$ In patients with metastatic disease, the goal of treatment is not only to treat localized areas of disease but also to target distant disease and prevent further dissemination of disease, which has typically been accomplished with systemic agents. As oncologists have not typically espoused the concurrent treatment of patients with brain radiation and systemic therapy due to the potential for neurotoxicity, the delivery of SRS rather than WBRT in selected patients has been favored as it subsequently allows the quicker initiation or resumption of systemic therapy.

There is, however, no report in the literature examining the timing of post-SRS systemic therapy (PoSST). While the importance of systemic therapy in managing metastatic patients is clear, the evaluation of the significance of systemic therapy before (pre-SRS systemic therapy, PrSST) and after SRS (PoSST) and the identification of an optimal time interval between SRS and PoSST may result in improved outcomes. Thus, the purpose of our study is not only to report our institutional experience, but also to assess the role of systemic therapy before and after SRS, and to evaluate the prognostic value of its timing after SRS.

\section{Methods and Materials}

We reviewed our institutional database for patients undergoing SRS for brain metastases between 2007 and 2013 and found 241 consecutively treated patients. Of these, 96 patients had primary lung cancer with 1-4 brain metastases; 6 patients with small cell lung cancer and 5 patients who expired $\leq 30$ days after SRS were excluded leaving 85 patients with NSCLC for analysis in this study. All patients had ECOG PS $\leq 3$ and were treated with a linear accelerator (LINAC) utilizing the BrainLAB system (BrainLAB Inc, Munich, Germany).

All procedures followed were in accordance with the ethical standards of the responsible committee on human experimentation (institutional and national) and with the Helsinki Declaration of 1975, as revised in 2008. The risks, benefits, and logistics of SRS were discussed with all patients after which informed consent for SRS was obtained by the radiation oncology team. The prescription dose was based on the target volume, any history of prior irradiation, and the proximity of critical organs at risk. The treatment plans were normalized so that the minimum tumor dose was the prescription dose. In most cases plans were optimized such that $>99.5 \%$ of the tumor volume received $>99.5 \%$ of the prescription dose. Patients were generally seen 1 month after SRS and then 1-3 months thereafter. Follow-up information was obtained from the electronic medical record (EMR) or by contacting the patient's family in cases where patients were lost to follow-up.

For analysis, clinical factors were recorded including ECOG performance status (PS), whether the patient underwent metastectomy or WBRT prior to SRS, the histologic diagnosis, the systemic status of disease (SDS) at time of SRS, and the use and duration of pre- and post-SRS systemic therapy (PrSST and PoSST). PrSST included any systemic therapy given to the patient prior to SRS regardless of whether the patient had only local or distant disease. The SDS was designated as either pulmonary disease (one or both lungs with adjacent nodal involvement) or distant extrapulmonary disease. SRS treatment-related factors were also recorded and included number of lesions, target volume size, and prescription dose. Dates of primary diagnosis, first CNS disease, and PoSST initiation were recorded as well.

All statistical tests were performed utilizing SPSS V21.0 (SPSS Inc., Chicago, IL) with a level of significance at $\mathrm{p}=$ 0.05. Actuarial survival analysis was performed. Univariate analysis of the aforementioned variables was performed using log-rank and regression tests. Variables that were found to be significant were then entered into a multivariate survival analysis utilizing the Cox proportional hazards model. Subsequently, subset analysis of patients with synchronous metastases (diagnosed $\leq 2$ months from primary diagnosis) was performed.

\section{Results}

Patient demographics and treatment characteristics are shown in Table 1; a total of 85 patients were included in this study. PrSST and PoSST were given at the discretion of the medical oncologist. Before SRS, 41 patients received PrSST of which 21 patients had records accessible to us with documentation of the agents utilized. Of these, 15 received chemotherapy alone, 1 received targeted therapy alone, and 5 received both. After SRS, 41 patients received PoSST. Thirty-three patients received chemotherapy alone, 1 patient received targeted therapy alone, and 7 patients received both. Eighteen patients received both PrSST and PoSST, while 64 received either. Chemotherapy consisted of a number of different agents given in different combinations and included carboplatin, paclitaxel, pemetrexed, cisplatin, etoposide, and gemcitabine. Targeted agents included erlotinib and bevacizumab. Of the 20 patients with known and available EGFR status, only 1 was positive for the mutation. 
Table 1: Patient demographics and treatment characteristics

\begin{tabular}{|c|c|}
\hline No. patients & 85 \\
\hline Age, median & 65 years $(41-85)$ \\
\hline \multicolumn{2}{|l|}{ Gender } \\
\hline Male & 47 pts $(55 \%)$ \\
\hline Female & 38 pts $(45 \%)$ \\
\hline \multicolumn{2}{|l|}{ Race } \\
\hline White & 31 pts $(37 \%)$ \\
\hline Black & 27 pts $(32 \%)$ \\
\hline Hispanic & 23 pts $(27 \%)$ \\
\hline Asian & 3 pts $(4 \%)$ \\
\hline Missing & $1 \mathrm{pt}(1 \%)$ \\
\hline \multicolumn{2}{|l|}{ ECOG Performance Status } \\
\hline 0 & 35 pts $(41 \%)$ \\
\hline 1 & 16 pts $(19 \%)$ \\
\hline 2 & 5 pts $(6 \%)$ \\
\hline 3 & 5 pts $(6 \%)$ \\
\hline Missing & $24(28 \%)$ \\
\hline \multicolumn{2}{|l|}{ Histology } \\
\hline Adenocarcinoma & 56 pts $(66 \%)$ \\
\hline Squamous Cell Carcinoma & 11 pts $(13 \%)$ \\
\hline Poorly Differentiated & 14 pts $(17 \%)$ \\
\hline Other & 4 pts $(5 \%)$ \\
\hline \multicolumn{2}{|l|}{ Prior to SRS } \\
\hline Metastectomy & 22 pts $(26 \%)$ \\
\hline WBRT & 9 pts $(11 \%)$ \\
\hline Both & 4 pts $(5 \%)$ \\
\hline \multicolumn{2}{|l|}{ SRS Parameters } \\
\hline No. lesions & 135 \\
\hline 1 & 47 pts $(55 \%)$ \\
\hline 2 & 30 pts $(35 \%)$ \\
\hline 3 & 5 pts $(6 \%)$ \\
\hline 4 & 2 pts $(2 \%)$ \\
\hline 5 & 1 pt $(1 \%)$ \\
\hline Target Volume, median & $1.6 \mathrm{cc}(0.1-21.8)$ \\
\hline Target Volume, interquartile range & $0.5-4.8 \mathrm{cc}$ \\
\hline Prescription dose, median & 21 Gy (12-25) \\
\hline \multicolumn{2}{|l|}{ Systemic Status of Disease (SDS) at SRS } \\
\hline Pulmonary & 48 pts $(57 \%)$ \\
\hline Extrapulmonary & 34 pts $(40 \%)$ \\
\hline Not specified & 3 pts $(4 \%)$ \\
\hline Primary to CNS disease diagnosis interval, median & 2 months $(0-88)$ \\
\hline \multicolumn{2}{|l|}{ Pre-SRS Systemic Therapy (PrSST) } \\
\hline Received & 41 pts $(48 \%)$ \\
\hline Duration, median & 5 months $(1-60)$ \\
\hline \multicolumn{2}{|l|}{ Post-SRS Systemic Therapy (PoSST) } \\
\hline Received & 41 pts $(48 \%)$ \\
\hline Duration, median & 2 months (1-19) \\
\hline \multicolumn{2}{|l|}{ PrSST and PoSST } \\
\hline Received & 18 pts $(21 \%)$ \\
\hline \multicolumn{2}{|l|}{ SRS to PoSST Interval (SPI) in pts receiving PoSST } \\
\hline SPI, median & 32 days (1-252) \\
\hline SPI, interquartile range & 19-59 days \\
\hline $\mathrm{SPI} \leq 30$ days & 19 pts $(45 \%)$ \\
\hline SPI > 30 days & 23 pts $(55 \%)$ \\
\hline
\end{tabular}




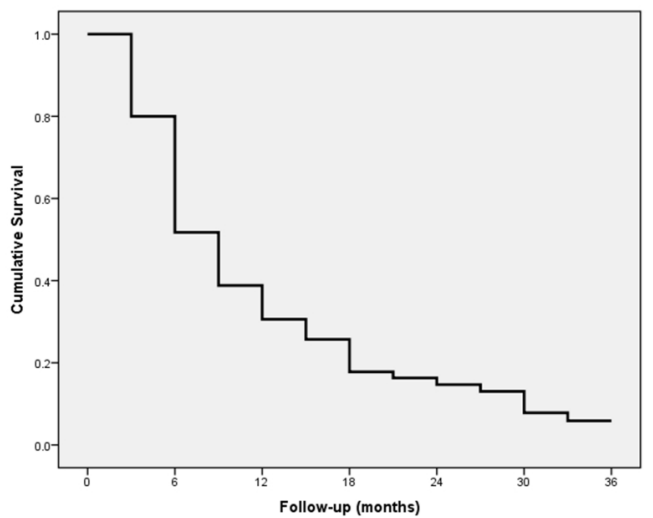

Figure 1: Actuarial survival for all patients

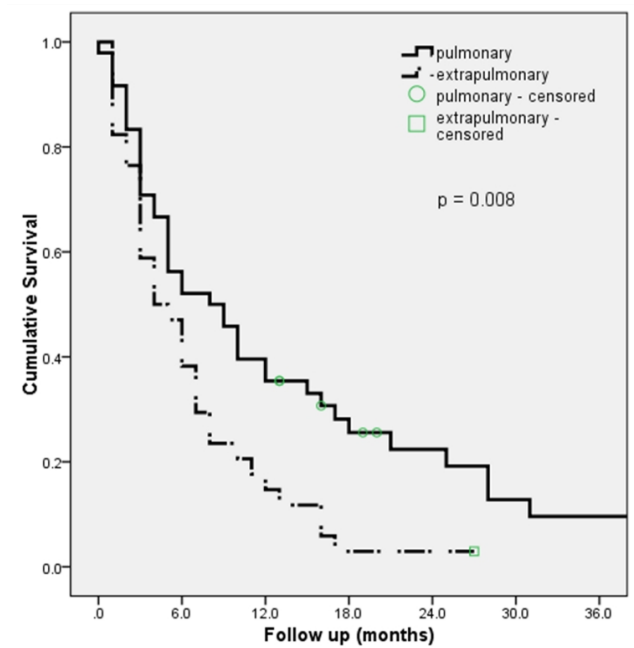

Figure 2: Kaplan Meier survival curve comparing systemic status of disease (extrapulmonary vs. pulmonary)

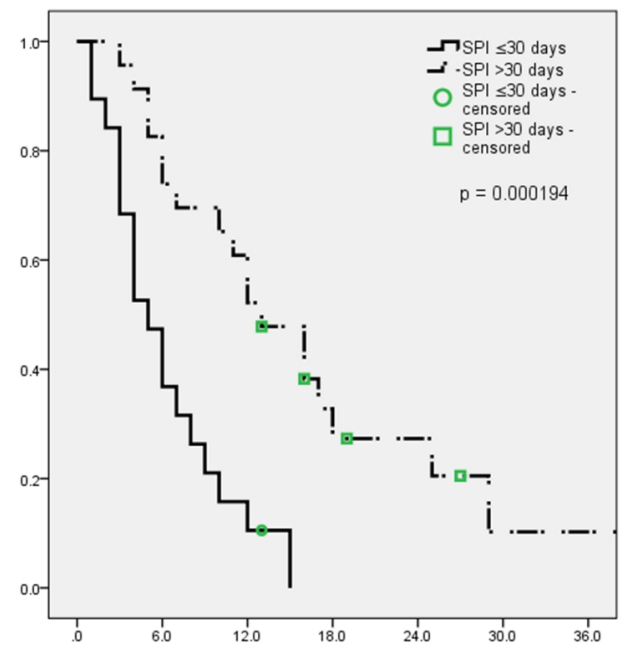

Figure 3: Kaplan Meier survival curve comparing the SRS to post-SRS systemic therapy interval (SPI)
At a median follow-up of 6 months (1-59), the median survival (MS) was 6.4 months. Figure 1 shows the actuarial survival for the cohort. The actuarial overall survival (OS) at 3, 6, 12, and 36 months was $80 \%, 52 \%$, $31 \%$, and $6 \%$, respectively. The median time interval between SRS and PoSST initiation (SPI) was 32 days. For further analysis, the SPI was divided into $\leq 30$ days and $>30$ days.

Table 2 shows significant predictors for survival based on univariate analysis. Age ( $p=0.092)$, race $(p=0.862)$, histology ( $p=0.180)$, and metastectomy ( $p=0.878)$ or WBRT $(\mathrm{p}=0.520)$ prior to SRS were not significant. SRS treatment factors including number of lesions ( $\mathrm{p}=$ $0.738)$, target volume ( $p=0.160)$, and prescription dose $(\mathrm{p}=0.150)$ were not significant. The primary-to-CNS disease interval $(\mathrm{p}=0.893)$ and the receipt of PrSST $(\mathrm{p}=$ 0.490 ) were not significant. Receipt of both PrSST and PoSST was not significant.

Significant clinical predictors by multivariate analysis are shown in Table 3. ECOG PS was not entered into this analysis due to the lack of data in approximately $1 / 3$ of patients. The use of any systemic therapy (PrSST or PoSST) as well as the use of PoSST wereremoved from this analysis because of the linear dependence of SPI on PoSST. Extrapulmonary disease was found to negatively predict for survival while an SPI $>30$ days was found to positively predict for survival. Figures $\mathbf{2}$ and $\mathbf{3}$ show Kaplan Meier survival curves comparing SDS and the SPI, respectively.

Given that the ECOG PS, SDS, and receipt of PrSST could potentially influence the significance of the SPI, log-rank tests were performed comparing SPI after stratifying by each of these factors. This revealed that SPI $>30$ days was prognostic in patients with ECOG PS $=0(\mathrm{p}=0.003)$ and regardless of SDS $(p=0.00049)$. SPI $>30$ days remained significant in both patients who received PrSST $(p=0.024)$ and in those who had not received $\operatorname{PrSST}(\mathrm{p}=0.003)$.

Thirty-nine (46\%) patients were found to have synchronous brain metastases (diagnosed at presentation or $\leq 2$ months of primary diagnosis). Analysis of these patients revealed that SDS did not significantly predict for survival by log-rank test ( $\mathrm{p}=$ 0.841). Even after further stratifying SDS into unilateral lung, bilateral lung, and extrapulmonary disease, SDS remained nonsignificant $(p=0.923)$. The receipt of PoSST continued to be prognostic $(p=0.020)$, and an SPI $>30$ days also continued to predict for improved survival $(\mathrm{p}=0.001)$. 
Table 2: Significant predictors for survival based on univariate analysis by log-rank test

\begin{tabular}{|c|c|c|c|}
\hline "Clinical Predictor & $\begin{array}{c}\text { Number of patients, } \\
N=96 \\
\end{array}$ & $\begin{array}{c}\text { Median Survival (months), } \\
\text { 95\% Confidence Interval }\end{array}$ & p-value \\
\hline $\begin{array}{l}\text { ECOG performance status } \\
0 \\
1 \\
2 \\
3 \\
\text { Missing }\end{array}$ & $\begin{array}{c}35(41 \%) \\
16(19 \%) \\
5(6 \%) \\
5(6 \%) \\
24(28 \%)\end{array}$ & $\begin{array}{l}9,2.4-15.6 \\
3,2.4-3.6 \\
3,0.9-5.1 \\
5,0-13.6\end{array}$ & 0.029 \\
\hline $\begin{array}{l}\text { Systemic Status of Disease (SDS) at SRS } \\
\text { Extrapulmonary Distant } \\
\text { Pulmonary }\end{array}$ & $\begin{array}{l}34(40 \%) \\
58(57 \%)\end{array}$ & $\begin{array}{c}4,1.6-6.4 \\
8,3.8-12.2\end{array}$ & 0.008 \\
\hline $\begin{array}{l}\text { Any Systemic Therapy } \\
\text { No } \\
\text { Yes }\end{array}$ & $\begin{array}{l}21(25 \%) \\
64(75 \%)\end{array}$ & $\begin{array}{l}3,1.9-4.1 \\
6,4.0-8.0\end{array}$ & 0.050 \\
\hline $\begin{array}{l}\text { Post-SRS Systemic Therapy (PoSST) } \\
\text { No } \\
\text { Yes }\end{array}$ & $\begin{array}{l}41(48 \%) \\
41(48 \%)\end{array}$ & $\begin{array}{c}3,1.9-4.1 \\
9,4.8-13.2\end{array}$ & 0.039 \\
\hline $\begin{array}{l}\text { SRS to PoSST Interval (SPI) in pts } \\
\text { receiving PoSST, } n=42 \\
\leq 30 \text { days } \\
>30 \text { days }\end{array}$ & $\begin{array}{l}19(45 \%) \\
23(55 \%) \\
\end{array}$ & $\begin{array}{c}5,2.9-7.1 \\
13,8.5-17.5 \\
\end{array}$ & 0.00019 \\
\hline
\end{tabular}

Table 3: Multivariate analysis of clinical predictors for survival by Cox Regression in patients who received post-SRS systemic therapy

\begin{tabular}{lcc}
\hline \hline Clinical Predictor & HR, 95\% Confidence Interval & $\boldsymbol{p}$-value \\
\hline Systemic Status of Disease (SDS) & $2.13,1.05-4.32$ & 0.036 \\
SRS to PSST Interval (SPI) & $0.28,0.13-0.62$ & 0.002 \\
\hline \hline
\end{tabular}

\section{Discussion}

Studies examining outcomes after SRS for brain metastases from lung cancer primaries have revealed MS times ranging from 3-15 months ${ }^{17,25}$ depending on varying prognostic factors. We found a MS of 6.4 months in our cohort, which is on the lower end of the survival spectrum found in the literature. However, our inclusion criteria were broader than those in a number of these studies, and included patients with varying performance statuses and various resection statuses for the lesion of interest.

In patients with multiple metachronous brain metastases from NSCLC treated with SRS, retrospective reviews have found median survivals of 7-11 months. ${ }^{17,18,21,23,24,26}$ DiLuna et al. found a significant survival difference in patients treated with SRS for 1-3 brain metastases versus those with $\geq 4$; however, our study, along with those performed by Likhacheva et al., Jezierska et al., and Smith et al. found no difference in survival based on number of intracranial lesions. ${ }^{24,27}$ Additionally, intracranial lesion size did not impact survival in our study but was found to be significant in other studies. ${ }^{17,26}$
Performance status has also been utilized to predict survival in patients with brain metastases treated with SRS. While Li et al. did not find Karnofsky Performance Status (KPS) to predict survival, a number of other studies have. ${ }^{19,23,24,26,28}$ In the patients for which we had ECOG PS available, we found better PS to be prognostic. We also found limited extracranial disease at SRS to be significantly associated with improved survival, and this was similarly noted in other retrospective reviews. ${ }^{17,21,24,26,27}$ This makes sense as patients with distant diffuse disease would be expected to succumb to their illness faster than those with better systemic control.

In addition to reporting our institutional experience, the focus of this paper is to explore the significance of preand post-SRS systemic therapy (PrSST and PoSST). Furthermore, we wish to discuss our interesting findings concerning the timing of PoSST initiation. As the goal of metastatic disease treatment in patients with good PS remains administration of systemic agents, there has been a shift in recent years to deliver SRS rather than WBRT in selected patients thereby allowing the quicker administration of systemic therapy after addressing intracranial disease. 
Chemotherapy traditionally played a limited role in the treatment of brain metastases as the integrity of the blood-brain-barrier was thought to limit delivery of drugs to the site of brain metastases. ${ }^{8,29}$ However, the tumor-specific enhancing properties of agents used in CT and MR suggest that the BBB may not be completely intact in patients with established brain metastases. In patients who have not been heavily pretreated with chemotherapy, the responses of brain metastases generally have been similar to extracranially located tumors of like histology. ${ }^{9,29}$ Phase II trials have demonstrated that temozolomide, topotecan, and paclitaxel with WBRT elicit some intracranial response.

First-line combination chemotherapy consisting of paclitaxel/cisplatin and gemcitabine/vinorelbine/ carboplatin in patients with brain metastases from NSCLC have yielded intracranial responses similar to extracranial responses suggesting the utility of systemic therapy. ${ }^{30,31}$ Other radiosensitizing agents such as gefitinib, motexafin gadolinium, efaproxiral, and bromodeoxyuridine have also been studied with a potential improvement in local control.8,13 The anti-angiogenic agent bevacizumab is the first targeted agent that demonstrated superior efficacy over chemotherapy alone as first-line treatment of advanced non-squamous NSCLC patients. , $^{9,11}$

While there have been a number of prospective trials examining the impact of systemic therapy in combination with WBRT $^{12}$, there is limited data available on the use of systemic therapy after SRS despite its widespread use in the last decade. DiLuna et al. reviewed 334 patients with intracranial disease from NSCLC, breast cancer, and melanoma who underwent SRS as initial therapy. ${ }^{27}$ In the subgroup of patients with $\geq 4$ brain metastases, receipt of chemotherapy was associated with decreased survival but did not reach statistical significance $(\mathrm{p}=0.077)$.

In a phase III trial, Sperduto et al. compared WBRT and SRS alone to WBRT and SRS with temozolomide or erlotinib for NSCLC and 1-3 brain metastases. ${ }^{10}$ The MS times between the arms were not significantly different. Time to CNS progression and PS at 6 months was better in the WBRT and SRS arm, and grade 3-5 toxicity was significantly worse in the temozolomide and erlotinib arms ( $\mathrm{p}<0.001)$. They concluded that the addition of temozolomide or erlotinib to WBRT and SRS did not improve survival and possibly had a deleterious effect.

In our study, receipt of any systemic therapy (PrSST or PoSST) and the receipt of PoSST alone were prognostic. Conversely, receipt of PrSST alone or both PrSST and PoSST were not significant leading us to conclude that it was the PoSST that contributed more to improved survival vs. the PrSST. As patients who died within 30 days after SRS may have not received systemic therapy due to deteriorating PS, we did not include them in order to prevent them from confounding our analysis of the value of PoSST and its timing. Multivariate analysis showed the absence of extrapulmonary disease and an SRS-to-PoSST interval (SPI) $>30$ days to significantly improve survival. The latter is a new and interesting finding as conventional thinking has suggested initiation or resumption of systemic therapy as soon as possible after SRS in order to prevent systemic disease progression. SRS has also been favored in certain cases over intracranial resection because of the ability to resume systemic therapy more rapidly after radiation versus surgery. ${ }^{32}$ We initially thought that ECOG PS may impact this finding as patients with better PS may do better regardless of SPI; however SPI $>30$ days remained significant even when controlling for PS. Receipt of PrSST may have also impacted the significance of an SPI $>30$ days given that a)patients who had already received PrSST immediately prior to SRS may have been delayed in their initiation of PoSST as they already had systemic therapy onboard or b)patients who had received PrSST may have been escalated to $2^{\text {nd }}$ or $3^{\text {rd }}$ line treatments thus indicating an already worse prognosis. However, an SPI $>30$ days predicted for improved survival in both patients who had received PrSST and those who had not. Lastly, the extent of distant disease may have also impacted the significance of an SPI >30 days, as more advanced systemic disease would likely require quicker initiation of systemic therapy after SRS. However, our findings remained even after stratifying patients by SDS. This seems to suggest that the rapid initiation of chemotherapy after SRS could be detrimental, possibly due to an increase in neurological complications.

While a number of phase I/II trials were conducted to evaluate the safety and efficacy of SRS prior to its adoption as a treatment standard for 1-3 brain metastases in certain patients, limited data exists comparing the safety and efficacy of SRS with or without systemic therapy, and no data was found in the literature regarding the optimal timing of systemic therapy after SRS. Sperduto et al. demonstrated an increase in grade 3-5 toxicity in the drug arms but was underpowered to prove that these toxicities led to a decrease in survival. ${ }^{10}$

We also sought to determine whether epidermal growth factor receptor (EGFR) status impacted outcome given that EGFR inhibitors have been found to have an intracranial response. ${ }^{33}$ To do this, we utilized the EGFR status of the primary lesion which has been shown to be a good surrogate for the EGFR status of the brain metastasis. ${ }^{34}$ However, only 20 patients in our cohort had this information determined and available and only one of these patients had the mutation preventing any meaningful analysis.

Patients with synchronous intracranial metastases from NSCLC are also thought to represent a unique population, and there is evidence supporting aggressive local and systemic therapy for these patients. ${ }^{35,38}$ In our study, the subset of patients with synchronous metastases benefited from the addition of systemic therapy after SRS. Again, interestingly, these patients 
also seemed to benefit from delaying systemic therapy to $>30$ days after SRS. Prospective studies would need to be conducted in order to evaluate the optimal timing of PoSST initiation in all patients with NSCLC brain metastases.

A strength of this study is the large cohort of patients with primary NSCLC with brain metastases treated at a single institution by designated radiation oncology and neurosurgical teams over a period of six years. While there are a number of studies which have reported their experience with SRS, our work represents the first radiosurgery study to evaluate the prognostic value of the timing of PoSST initiation. Limitations of this study include those inherent to the retrospective nature of this review as well as the somewhat heterogeneous cohort of patients included in this study in regards to systemic therapy - various regimens were used at various time points and for different reasons depending on the medical oncologist. Additionally, we only had PS documented for approximately $2 / 3$ of our patients in this study via our EMR. A full dataset would have allowed better and more complete analysis of our findings. Also, due to follow-up data on a number of patients being obtained from the hospital EMR, neurologic toxicities from radiation therapy were not consistently and accurately documented preventing significant analysis of adverse late effects of radiosurgery and PoSST. This also prevented a meaningful comparison in neurologic toxicities in regards to SPI.

\section{Conclusion}

This study shows that the use of systemic therapy before and after SRS is beneficial in patients with NSCLC brain metastases. Our results also suggest that delaying the initiation of systemic therapy after SRS to $>30$ days may potentially improve survival, even in patients with synchronous metastases. This finding may have potentially been influenced by neurotoxicity after SRS. As there is a paucity of evidence regarding the timing of systemic therapy after SRS, further investigation is warranted to define the optimal SRS-PoSST interval.

\section{Conflict of interest}

The authors declare that they have no conflicts of interest. The authors alone are responsible for the content and writing of the paper.

\section{References}

1. Palmieri D. Central nervous system metastasis, the biological basis and clinical considerations. New York: Springer; 2012.

2. Davis FG, Dolecek TA, McCarthy BJ, et al. Toward determining the lifetime occurrence of metastatic brain tumors estimated from 2007 united states cancer incidence data. Neuro Oncol 2012;14:1171-7.

3. Patchell RA, Tibbs PA, Regine WF, et al. Postoperative radiotherapy in the treatment of single metastases to the brain: A randomized trial. JAMA 1998; 280:1485-9.

4. Patchell RA, Tibbs PA, Walsh JW, et al. A randomized trial of surgery in the treatment of single metastases to the brain. N Engl J Med 1990;322:494-500.

5. Andrews DW, Scott CB, Sperduto PW, et al. Whole brain radiation therapy with or without stereotactic radiosurgery boost for patients with one to three brain metastases: Phase iii results of the rtog 9508 randomised trial. Lancet 2004;363:1665-72.

6. Chang EL, Wefel JS, Hess KR, et al. Neurocognition in patients with brain metastases treated with radiosurgery or radiosurgery plus whole-brain irradiation: A randomised controlled trial. Lancet Oncol 2009;10:1037-44.

7. Tsao M, Xu W, Sahgal A. A meta-analysis evaluating stereotactic radiosurgery, whole-brain radiotherapy, or both for patients presenting with a limited number of brain metastases. Cancer 2012;118:2486-93.

8. Langer CJ, Mehta MP. Current management of brain metastases, with a focus on systemic options. J Clin Oncol 2005;23:6207-19.

9. Walbert T, Gilbert MR. The role of chemotherapy in the treatment of patients with brain metastases from solid tumors. Int J Clin Oncol 2009;14:299-306.

10. Sperduto PW, Wang M, Robins HI, et al. A phase 3 trial of whole brain radiation therapy and stereotactic radiosurgery alone versus wbrt and srs with temozolomide or erlotinib for non-small cell lung cancer and 1 to 3 brain metastases: Radiation therapy oncology group 0320. Int J Radiat Oncol Biol Phys 2013;85:1312-18.

11. Lombardi G, Di Stefano AL, Farina P, et al. Systemic treatments for brain metastases from breast cancer, non-small cell lung cancer, melanoma and renal cell carcinoma: An overview of the literature. Cancer Treat Rev 2014;40(8):951-9.

12. Zimmermann S, Dziadziuszko R, Peters S. Indications and limitations of chemotherapy and targeted agents in non-small cell lung cancer brain metastases. Cancer Treat Rev 2014;40:716-22.

13. Dawe DE, Greenspoon JN, Ellis PM. Brain metastases in non-small-cell lung cancer. Clin Lung Cancer 2014;15:249-57.

14. Fidler IJ. The biology of brain metastasis: Challenges for therapy. Cancer J 2015;21:284-93. 
15. Hu J, Kesari S. Strategies for overcoming the blood-brain barrier for the treatment of brain metastases. CNS Oncol 2013;2:87-98.

16. Puhalla S, Elmquist W, Freyer D, et al. Unsanctifying the sanctuary: Challenges and opportunities with brain metastases. Neuro Oncol 2015;17:639-51.

17. Kim YS, Kondziolka D, Flickinger JC, et al. Stereotactic radiosurgery for patients with nonsmall cell lung carcinoma metastatic to the brain. Cancer 1997;80:2075-83.

18. Williams J, Enger C, Wharam M, et al. Stereotactic radiosurgery for brain metastases: Comparison of lung carcinoma vs. Non-lung tumors. J Neurooncol 1998;37:79-85.

19. Li B, Yu J, Suntharalingam M, et al. Comparison of three treatment options for single brain metastasis from lung cancer. Int J Cancer 2000;90:37-45.

20. Serizawa T, Ono J, Iichi T, et al. Gamma knife radiosurgery for metastatic brain tumors from lung cancer: A comparison between small cell and non-small cell carcinoma. J Neurosurg 2002;97:484-88.

21. Zabel A, Milker-Zabel S, Thilmann C, et al. Treatment of brain metastases in patients with non-small cell lung cancer (nsclc) by stereotactic linac-based radiosurgery: Prognostic factors. Lung Cancer 2002;37:87-94.

22. Noel G, Medioni J, Valery CA, et al. Three irradiation treatment options including radiosurgery for brain metastases from primary lung cancer. Lung Cancer 2003;41:333-43.

23. Kress MA, Oermann E, Ewend MG, et al. Stereotactic radiosurgery for single brain metastases from non-small cell lung cancer: Progression of extracranial disease correlates with distant intracranial failure. Radiat Oncol 2013;8:64.

24. Jezierska D, Adamska K, Liebert W. Evaluation of results of linac-based radiosurgery for brain metastases from primary lung cancer. Rep Pract Oncol Radiother 2014;19:19-29.

25. Smith TR, Lall RR, Lall RR, et al. Survival after surgery and stereotactic radiosurgery for patients with multiple intracranial metastases: Results of a single-center retrospective study. J Neurosurg 2014:1-7.

26. Likhacheva A, Pinnix CC, Parikh NR, et al. Predictors of survival in contemporary practice after initial radiosurgery for brain metastases. Int J Radiat Oncol Biol Phys 2013;85:656-61.

27. DiLuna ML, King JT, Jr., Knisely JP, et al. Prognostic factors for survival after stereotactic radiosurgery vary with the number of cerebral metastases. Cancer 2007;109:135-45.

28. Yomo S, Hayashi M. Upfront stereotactic radiosurgery in patients with brain metastases from small cell lung cancer: Retrospective analysis of 41 patients. Radiat Oncol 2014;9:152.

29. Davey P. Brain metastases: Treatment options to improve outcomes. CNS Drugs 2002;16:325-38.

30. Bernardo G, Cuzzoni Q, Strada MR, et al. First-line chemotherapy with vinorelbine, gemcitabine, and carboplatin in the treatment of brain metastases from non-small-cell lung cancer: A phase ii study. Cancer Invest 2002;20:293-302.

31. Cortes J, Rodriguez J, Aramendia JM, et al. Front-line paclitaxel/cisplatin-based chemotherapy in brain metastases from non-small-cell lung cancer. Oncology 2003;64:28-35.

32. Lippitz B, Lindquist C, Paddick I, et al. Stereotactic radiosurgery in the treatment of brain metastases: The current evidence. Cancer Treat Rev 2014;40:48-59.

33. Luo D, Ye X, Hu Z, et al. Egfr mutation status and its impact on survival of chinese non-small cell lung cancer patients with brain metastases. Tumour Biol 2014;35:2437-44.

34. Jamal-Hanjani M, Spicer J. Epidermal growth factor receptor tyrosine kinase inhibitors in the treatment of epidermal growth factor receptor-mutant non-small cell lung cancer metastatic to the brain. Clin Cancer Res 2012;18:938-44.

35. Arrieta O, Villarreal-Garza C, Zamora J, et al. Long-term survival in patients with non-small cell lung cancer and synchronous brain metastasis treated with whole-brain radiotherapy and thoracic chemoradiation. Radiat Oncol 2011;6:166.

36. Funai K, Suzuki K, Sekihara K, et al. Five-year tumor-free survival after aggressive trimodality therapy for $\mathrm{t} 3 \mathrm{n} 0 \mathrm{~m} 1 \mathrm{~b}$ non-small cell lung cancer with synchronous solitary brain metastasis. Gen Thorac Cardiovasc Surg 2012;60:370-2.

37. Gray PJ, Mak RH, Yeap BY, et al. Aggressive therapy for patients with non-small cell lung carcinoma and synchronous brain-only oligometastatic disease is associated with long-term survival. Lung Cancer 2014;85:239-44.

38. Griffioen GH, Toguri D, Dahele M, et al. Radical treatment of synchronous oligometastatic non-small cell lung carcinoma (nsclc): Patient outcomes and prognostic factors. Lung Cancer 2013;82:95-102. 